\title{
Distinguishing between Directional Guidance and Motility Regulation in Neuronal Migration
}

\author{
Michael Ward, ${ }^{1}$ Corey McCann, ${ }^{1}$ Michael DeWulf, ${ }^{2}$ Jane Y. Wu, ${ }^{2}$ and Yi Rao ${ }^{1}$ \\ Departments of ${ }^{1}$ Anatomy and Neurobiology and ${ }^{2}$ Pediatrics and Molecular Biology and Pharmacology, Washington University School of Medicine, \\ St. Louis, Missouri 63110
}

\begin{abstract}
Although neuronal migration is an essential process in development, how neural precursors reach their final destination in the nervous system is not well understood. Secreted molecules that are known to be involved in axon guidance are likely to play important roles in regulating neuronal migration, but an important issue that remains unclear is whether such molecules act as directional guidance cues or as motility regulators in neuronal migration. The secreted protein Slit was initially suggested to be a repellent for migrating neurons (Wu et al., 1999). However, it was concluded recently that Slit plays an inhibitory rather than a repulsive role in neuronal migration (Mason et al., 2001). We have developed a series of assays that allow us to differentiate between repulsive and inhibitory effects of secreted molecules, and we demonstrate that Slit is a repellent capable of reversing the direction of neurons migrating either in culture or in their native pathways. We also show that although Slit reduces migratory speed under certain conditions, it can function as a repellent without concurrent inhibition of neuronal migration. This is the first study to clearly demonstrate that migrating neurons can be directionally guided by secreted molecules. These findings provide a basis to understand the physiological roles of secreted molecules in the developing nervous system and have implications on how they could be applied therapeutically. Our results also indicate that it should be possible to determine the specific action of other molecules as directional guidance cues or as motility regulators of cell migration.
\end{abstract}

Key words: cell migration; neuronal migration; axon guidance; guidance cues; Slit; motility

\section{Introduction}

The migration of neurons is an important feature of neural development, and defects in neuronal migration have been shown to cause a number of human diseases (Hatten and Mason, 1990; Rakic, 1990; Hatten and Heintz, 1998; Rice and Curran, 2001; Ross and Walsh, 2001). Thus, studies of the cellular and molecular mechanisms that control neuronal migration are important to further our understanding of neural development and the etiology of neurological diseases.

Signaling molecules including netrins, ephrins, semaphorins, Slits, and the chemokine stromal cell-derived factor have been implicated in controlling neuronal migration (Colamarino and Tessier-Lavigne, 1995; Kolodkin, 1996; Flanagan and Vanderhaeghen, 1998; Raper, 2000; Klein, 2001; Wong et al., 2002; Zhu et al., 2002). A number of these same molecules play important and well characterized roles in axon guidance. However, it is unclear whether migrating neurons and axons interpret these signals in the same way. In particular, although it is established that axons can be directionally guided by secreted molecules (Song et al., 1998), no direct evidence of directional guidance of neuronal migration exists.

In principle, a specific extracellular molecule can change ei-

Received Nov. 8, 2002; revised March 21, 2003; accepted April 3, 2003.

We are grateful to support from the National Institutes of Health (to Y.R.), NiA R01AG17518 (to J.Y.W.), the Klingenstein Foundation (to Y.R.), and the Medical Scientist Training Program (to M.W. and C.M.).

Correspondence should be addressed to either of the following: Dr. Jane Y. Wu, Department of Pediatrics and Molecular Biology and Pharmacology, Washington University School of Medicine, Box 8108, 660 South Euclid Avenue, St. Louis, M0 63110, E-mail: jwu@pcg.wustl.edu; or Dr. Yi Rao, Department of Anatomy and Neurobiology, Washington University School of Medicine, Box 8108, 660 South Euclid Avenue, St. Louis, M0 63110, E-mail: raoyi@thalamus.wustl.edu.

Copyright $\odot 2003$ Society for Neuroscience $\quad$ 0270-6474/03/235170-08\$15.00/0 ther the motility of a migrating cell or its migratory direction. These molecules can alter cell motility when they are present either in a uniform concentration or in a concentration gradient. However, a molecule can change the direction of a migrating cell only when it is present in a concentration gradient. It is important to note that: (1) cell motility and migratory direction are two different processes; and (2) although the activity of a molecule in its regulation of cell motility can be correlated with its guidance of migratory direction, this is not necessarily always the case. For example, although Slit is a repellent of axons in a number of regions of the nervous system (a negative effect) (Bashaw and Goodman, 1999; Brose et al., 1999; Kidd et al., 1999; Li et al., 1999; Nguyen Ba-Charvet et al., 1999; Bashaw et al., 2000; Fricke et al., 2001; Bagri et al., 2002; Hutson and Chien, 2002; Plump et al., 2002; Wong et al., 2002), it can also promote axon branching (a positive effect) (Wang et al., 1999). In the case of axons from the spinal cord of Xenopus, it was shown that Slit could both promote the outgrowth of these axons as well as repel them (Stein and Tessier-Lavigne, 2001). Therefore, a priori predictions of the effects of negative factors (i.e., correlated inhibition and repulsion) and positive factors (i.e., correlated motility promotion and attraction) may not be valid and can lead to oversimplification of the underlying mechanisms used in molecular control of cell migration.

Although some molecules may have more than one effect, it is important to distinguish between repulsion and inhibition of cell motility (and between attraction and promotion of cell motility). The implications of each of these effects are different in the context of both development and potential therapeutic applications. For example, a repellent can guide neuronal migration by directing a cell to a specific (and possibly distant) destination after 
being secreted from its source. In contrast, an inhibitor can only prevent a cell from approaching its source and thereby prevent a cell from entering into certain regions of the nervous system. In principle, a repellent could be used to guide transplanted stem cells to a target region if the repellent is strategically placed behind the transplanted cells, whereas an inhibitor cannot be used in the same manner. In contrast, an inhibitor could be used to restrain metastatic tumor cells if it is applied over these cells or on their pathways, whereas a repellent would have to be used differently because a repellent would turn tumor cells to another site without immobilizing them.

The Slit family of secreted proteins is an important factor in the development of both invertebrates and vertebrates (NüssleinVolhard et al., 1984; Rothberg et al., 1988; Rothberg and Artavanis-Tsakonas, 1992; Brose et al., 1999; Kidd et al., 1999; Li et al., 1999; Whitford et al., 2002; Wong et al., 2002). The suggestion that Slit is a repellent of migrating neurons was based on results from the coculture of neuronal explants with a human embryonic kidney (HEK) cell line secreting the Slit protein $(\mathrm{Hu}$, 1999; Wu et al., 1999; Zhu et al., 1999). It was observed in these experiments that more neurons migrate into the quadrant distal to the Slit source than the quadrant proximal to the Slit source (Hu, 1999; Wu et al., 1999; Zhu et al., 1999). However, such changes in cell distribution could be caused by Slit-induced inhibition or by Slit-induced repulsion. It has been reported recently that Slit functions only as an inhibitor, but not as a repellent, of migrating neurons (Mason et al., 2001). When Slit was applied to the medium uniformly around neuronal explants, the number of neurons migrating out of the explants was significantly reduced (Mason et al., 2001). The primary effect of Slit was, therefore, controversial. Using both in vitro and in vivo assays, we have now demonstrated that Slit is primarily a repellent of migrating neurons. We have found that Slit can slow down but not stop migrating neurons when presented in a uniform concentration. We also show that Slit can function as a repellent without causing inhibition of overall cell motility, and that the primary role of Slit is repulsion when presented in a concentration gradient. These studies provide definitive evidence that the primary effect of Slit in neuronal migration is a repulsive one and suggest generally applicable approaches to determine the roles of other extracellular molecules in neuronal migration.

\section{Materials and Methods}

Dissection and culture of anterior subventricular zone explants. Anterior subventricular zone (SVZa) explants were dissected from the rostral migratory stream (RMS) of postnatal day 2 (P2)-P5 rat brains as described previously (Wu et al., 1999). Briefly, coronal sections of the caudal half of the olfactory bulb were made with a tungsten needle, and the RMS was identified by its translucent appearance and dissected out. This tissue was used to make small explants (200-400 $\mu \mathrm{m}$ in diameter), which were suspended in a 2:2:1 matrigel:collagen:DMEM mixture. Explants were then cultured in DMEM plus $10 \%$ heat-inactivated FCS at $37^{\circ} \mathrm{C}$ in a $5 \%$ $\mathrm{CO}_{2}$ incubator for $24 \mathrm{hr}$.

Time-delayed coculture of HEK aggregates with SVZa explants. The generation of HEK-293 cells expressing mouse Slit-1 was described previously (Wu et al., 1999). Aggregates of Slit-secreting cells or HEK control cells were prepared using a hanging drop method. After the initial $24 \mathrm{hr}$ incubation of SVZa explants, media were removed from the matrigel/ collagen pad and a 400 - to $600-\mu \mathrm{m}$-wide cell aggregate was placed next to the explant. Additional matrigel/collagen was added to the top of the cell aggregate/explant and allowed to harden, followed by the addition of DMEM plus 10\% FCS. Explants were photographed using an upright microscope (Olympus, Tokyo, Japan) and CoolSnap HQ camera (Pho- tometrics) at the time of aggregate placement $(0 \mathrm{hr})$ and $24 \mathrm{hr}$ after aggregate placement. Results were quantified as follows: for each explant, all SVZa cell bodies that migrated beyond the visibly thickened edge of the explant were marked. The $X, Y$ coordinates of each cell in relation to the center of the explant per edge of the cell aggregate were recorded and used to sort cells into sampling regions.

Time-lapse imaging of migrating SVZa neurons. Explant culture and cell aggregate placement were performed as described previously, except for the following modifications: 1-2 hr before cell aggregate placement, DMEM culture medium was replaced with HEPES-buffered L-15 plus $10 \%$ FCS (overlaid with light mineral oil to prevent evaporation). Timelapse microscopy was then performed using an upright microscope (Olympus) and CoolSnap HQ camera equipped with a heated stage. The same SVZa neurons were tracked both before and after cell aggregate placement. Turning was quantified as follows: because the time in which cells turn in response to Slit aggregate placement is a factor of aggregate size, distance of aggregate to explant, and gelling time of the collagen/ matrigel mixture, the vector diagrams used in quantification were obtained by measuring the average angle of migration of these neurons (relative to aggregate location) for the hour preceding Slit/control aggregate placement and for the last hour of recording after Slit/control aggregate placement. Each vector diagram represents cells measured during the same time-lapse experiment.

Brain slice migration assay. P2-P5 rat brains were embedded in 4\% low melting point agarose, and $200-\mu \mathrm{m}$-thick sagittal sections were obtained at $4^{\circ} \mathrm{C}$ using a vibratome. These slices were placed on filters $(0.45 \mu \mathrm{m}$; Millipore, Bedford, MA) in a minimal amount of DMEM plus $10 \%$ FCS, and a single DiI crystal was placed at the genu of the RMS. Slices were cultured for $12 \mathrm{hr}$ and photographed. Then, a Slit-secreting aggregate or control cell aggregate was placed at the most rostral portion of the RMS and slices were cultured for an additional $12 \mathrm{hr}$, followed by another round of photography.

Conditioned media. Conditioned media were obtained from Slitsecreting cells or HEK control cells. Cells of $80 \%$ confluence were cultured in DMEM plus 10\% FCS for $24 \mathrm{hr}$, and their media were collected.

Cell speed analysis. For studies using time-lapse microscopy, conditioned media were collected as described previously and then concentrated 20-30 times via centrifugation with a filter (Centricon). The media were then diluted 1:20 in L-15 plus 10\% FCS, which was added to neurons. Neurons were randomly selected, and the distance that these cells migrated during the course of each $5 \mathrm{hr}$ treatment was measured using Metamorph (Universal Imaging, West Chester, PA), thus yielding cell speed. When neurons showed appreciable changes in migratory direction during this recording session $\left(>20^{\circ}\right)$, additional waypoints were marked along their migratory path, allowing for a more precise measurement of distance/time. For studies involving speed measurements before and after Slit aggregate placement, explants were cultured as described in Figure 3. Twenty-four neurons were selected on the following criteria: (1) that they turn in response to Slit aggregate placement, and (2) that they can be followed throughout the course of the entire experiment. The position of each of these cells (specifically the cell body) was marked at the beginning and end of each recording session, and the distance between these two points was measured. Because cells almost always slow down during the turning process itself after Slit aggregate placement, the speed of individual cells was measured after the turning of individual cells was complete. This resulted in a standard duration of speed measurement for each cell before the addition of Slit ( $4 \mathrm{hr})$ and after Slit-induced turning (4 hr).

\section{Results}

Changes in the distribution of migrating neurons in a timedelayed coculture assay

To determine whether Slit functions as a repellent or an inhibitor, we designed a new time-delayed coculture assay (Fig. 1a). In our previous coculture assays, explants from the SVZa were cultured at a distance from an aggregate of HEK cells secreting Slit (Wu et al., 1999; Zhu et al., 1999). In these previous assays, SVZa neurons 
have been exposed to Slit from the beginning of the coculture. When the distribution of SVZa neurons in the proximal quadrant is compared with the distal quadrant, differences can be interpreted as evidence for either repulsion ( $\mathrm{Wu}$ et al., 1999; Zhu et al., 1999) or inhibition (Mason et al., 2001). In the new time-delayed assay, we first cultured an SVZa explant in a matrigel/collagen mixture, and only after a time delay of $24 \mathrm{hr}$ did we place an aggregate of control or Slit-secreting HEK cells at a distance to the SVZa explant. Pictures were taken at two different time points, immediately after or $24 \mathrm{hr}$ after the placement of the HEK aggregate in the culture.

This time-delayed coculture assay allows us to obtain evidence concerning whether Slit can function as a repellent or only as an inhibitor, because each effect makes different predictions of the experimental outcome (Fig. 1a). In the presence of a control aggregate, the number of neurons should increase uniformly on all sides of the explant after aggregate placement (Fig. 1a, top). If Slit is solely an inhibitor, the number of neurons on the proximal side of the explant (Fig. $1 c$, sampling regions I-III) should either increase or remain the same (Fig. $1 a$, middle), because the presence of Slit is predicted to inhibit neuronal migration in a nondirectional manner. However, if Slit acts as a repellent, the number of neurons on the proximal side of the explant should decrease as cells move away from the source of Slit (Fig. 1a, bottom).

We observed that at $24 \mathrm{hr}$ after placement of the control aggregate, the number of neurons on the proximal side of the explant increased compared with the number of neurons present at the time of the aggregate placement (Fig. 1b, top). However, $24 \mathrm{hr}$ after the placement of the Slit aggregate, the number of neurons on the proximal side of explants was dramatically reduced compared with the number of neurons present at the time of the aggregate placement (Fig. 1b, bottom). Note the changes in distribution of both individual cells and cells involved in chain migration, indicating that cells involved in both of these forms of migration respond to Slit (Fig. $1 b$, bottom).

To quantify the above findings, the number of neurons in defined sampling regions (Fig. 1c) was determined both before and $24 \mathrm{hr}$ after aggregate placement. Twenty-four hours after placement of the control aggregate, a statistically significant increase in the number of neurons in all sampling regions was observed (Fig. $1 d$ ) (paired $t$ test; $p<0.005 ; n=30$ explants). Similarly, a statistically significant increase in the number of neurons in sampling regions $\mathrm{V}$ and VI was observed $24 \mathrm{hr}$ after the placement of the Slit-secreting aggregates (Fig. 1d) (paired $t$ test; $p<0.05 ; n=36$ explants). However, a statistically significant decrease in the number of neurons in sampling regions I and II was observed $24 \mathrm{hr}$ after the placement of the Slit aggregates (Fig. 1d) (paired $t$ test; $p<0.005 ; n=36$ explants). There was no statistically significant difference in the number of neurons in
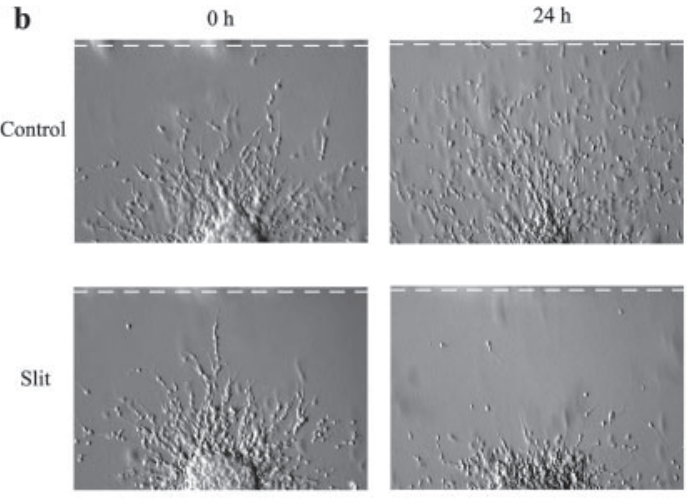

d

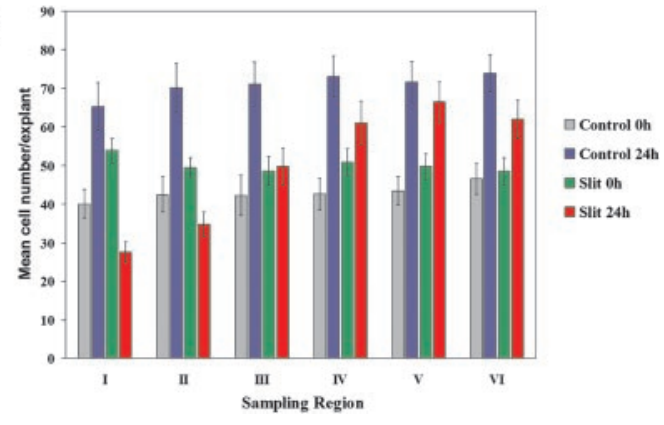

Figure 1. Time-delayed coculture assay and the effect of Slit. $a$, Diagram of the experimental design and predicted distributions meurons. Explants were first cultured for $24 \mathrm{hr}$ in the absence of cell aggregates (proximal neurons shown in red). The photographed at 0 and $24 \mathrm{hr}$. Individual cells were counted at both time points and assigned to the sampling regions shown in $c$. For each experimental group, $n>30$ explants. Error bars represent SEM.

sampling regions III and IV after Slit aggregate placement. These results are consistent with the idea that Slit can function as a repellent.

\section{Changes in the direction of neuronal migration when presented with a Slit source}

To directly characterize the response of migrating SVZa neurons to Slit, we performed time-lapse microscopy of individual SVZa neurons before and after the placement of a Slit-secreting aggregate or control aggregate (Fig. 2a). SVZa explants were cultured for $24 \mathrm{hr}$ before the placement of aggregates to allow for individual SVZa neurons to migrate out of the explants. The migratory behavior of these neurons was followed before and after the placement of the aggregates.

Whereas most neurons did not change direction after placement of the control aggregates (Fig. $2 a$, top), the majority of neurons in the proximal quadrant reversed their direction when exposed to Slit aggregates (Fig. $2 a$, bottom). To quantify the results from the time-lapse images, 20 neurons were randomly chosen from each experiment and tracked before and after aggregate placement (Fig. 2b). It was found that most neurons did not noticeably change direction $10 \mathrm{hr}$ after the placement of control aggregates. However, $85 \%$ of cells that were migrating toward the Slit aggregates at time 0 changed their direction and migrated away from the Slit aggregate $10 \mathrm{hr}$ later. These results provide direct evidence that Slit is a repellent. 
$\mathbf{a}$
$-1$
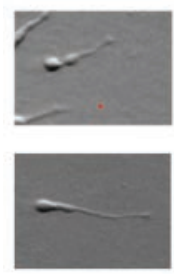

b
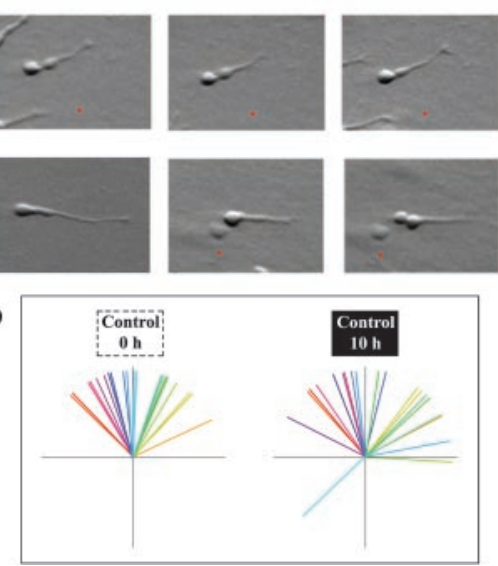

Time (h)

2
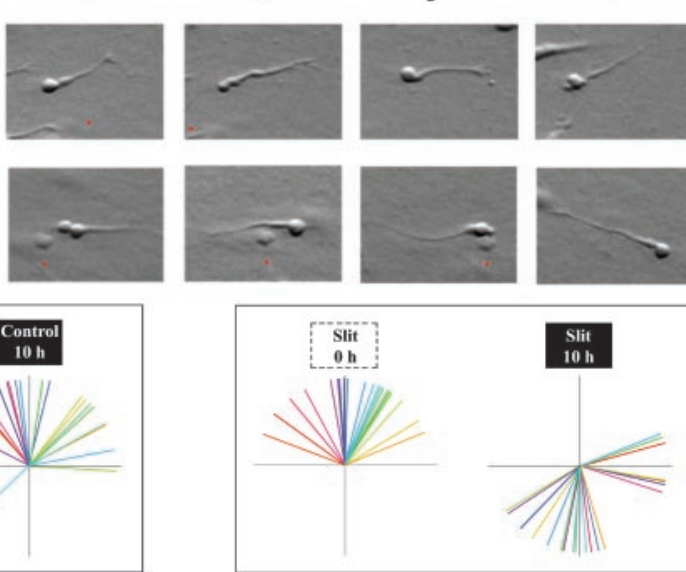

Figure 2. Neuronal repulsion by a gradient of Slit. $a$, Explants were cultured alone for $24 \mathrm{hr}$ before being cocultured with either Slit or control aggregates. Time-lapse microscopy was used to track neuronal migration. The Slit/control aggregates were placed on the right. Red dots illustrate stationary points in the pictures. $b$, Direction of neuronal migration before and after Slit/control aggregate placement. Time-lapse microscopy was used to track 20 neurons in each experimental group. Each colored line represents the angle of migration of an individual neuron in relation to the center of the explant and location of the aggregates. The same color is used to depict a neuron both before and after aggregate placement. Each pair of vector diagrams represents neurons from a single time-lapse experiment.

$\mathbf{a}$
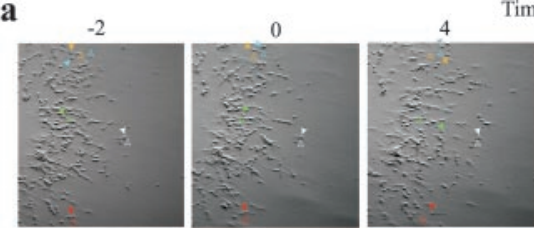

Time (h)
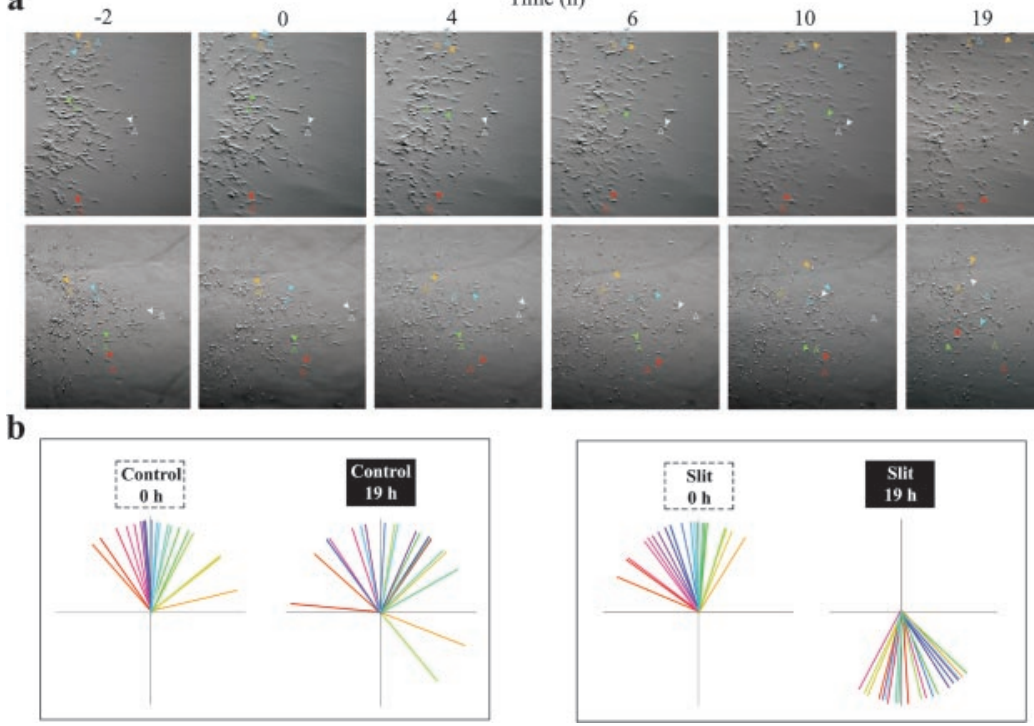

Figure 3. Neuronal repulsion by Slit after explant removal. $a$, Explants were cultured as described in Figure 2, except that the explants were dissected away after individual neurons had migrated out and replaced with a collagen/matrigel mixture before imaging. Different colored arrowheads indicate the location of individual neurons in each frame. Open arrowheads indicate the position of each neuron at the time of aggregate placement. $b$, The same directional analysis of SVZa migration described in Figure 2 applied to neuronal migration in the absence of an explant. Each pair of vector diagrams represents neurons from a single time-lapse experiment.

\section{Independence of repulsion by Slit from factors present in the explants}

It has been reported that a glial cell-derived factor was secreted by SVZa explants, and that Slit could only function as a repellent when the glial factor was present (Mason et al., 2001). We have also found that SVZa neurons out of the explants change their direction and migrate back into the explants when exposed to a uniform application of Slit (see Fig. 5). Therefore, it is important to determine whether the turning behavior of SVZa neurons observed in Figure 2 is also dependent on the presence of the explants.

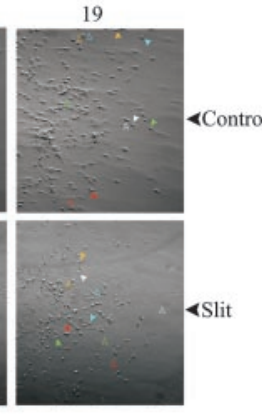

To determine whether individual SVZa neurons are repelled by Slit in the absence of the intact explants or glial cells in the explants, we removed the explants after SVZa neurons migrated out of the explants. We have shown that only neurons migrate out of the explants, whereas glial cells stay within the explants (Wu et al., 1999; Zhu et al., 1999). Thus, after explant removal, neither intact SVZa explants nor glial cells are present in the cultures. We then added aggregates and used time-lapse microscopy to observe the behavior of SVZa neurons. It was found that the presence of an explant was not required for Slit-mediated turning of migrating SVZa neurons (Fig. 3a). Whereas the majority of cells did not change direction after placement of control aggregates, placement of Slit aggregates caused SVZa neurons to change their migratory direction (Fig. 3b). These results indicate that individual SVZa neurons can be repelled by Slit in the absence of an intact SVZa explant.

\section{Directional changes of SVZa neurons migrating in situ by Slit}

Although the above results show that Slit can repel SVZa neurons when neurons migrate in collagen and matrigel cultures, it is not known whether Slit can repel SVZa neurons migrating in their native pathway, the RMS (Luskin, 1993; Lois and AlvarezBuylla, 1994). Previous results have only shown that Slit can prevent the migration of SVZa neurons into the RMS when an aggregate is placed on top of the RMS ( $\mathrm{Wu}$ et al., 1999), but it has not been demonstrated that Slit can repel migrating SVZa neurons in situ.

To rule out the possibility that Slit can repel SVZa neurons in collagen and matrigel cultures but can only exert an inhibitory effect on SVZa neurons migrating in the native pathway, we used the lipophilic dye DiI to label SVZa neurons in postnatal rat brain slices (Fig. 4a). Twelve hours after the insertion of a DiI crystal into the RMS, numerous labeled SVZa neurons migrated rostrally toward the olfactory bulb (Fig. 4b, $0 \mathrm{hr}$ ). At this time, either Slit or control aggregates were placed on top of the RMS at a position rostral to the DiI-labeled neurons. Slices were then cultured for an additional $12 \mathrm{hr}$. Twelve hours after the placement of control aggregates, an increased number of labeled neurons rostral to the DiI crystal were observed (Fig. 4b, top right). In contrast, the number of labeled neurons rostral to the DiI crystal after the placement of Slit aggregates was clearly decreased (Fig. $4 b$, bottom right) compared with the number of labeled neurons present at the time of aggregate placement (Fig. $4 b$, bottom left). To quantitate these findings, the number of labeled neurons on the proximal side of the DiI crystal (i.e., the side facing the control/Slit aggregate) was counted both before 
$\mathbf{a}$

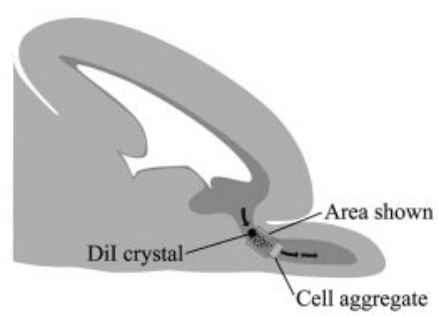

b

$0 \mathrm{~h}$
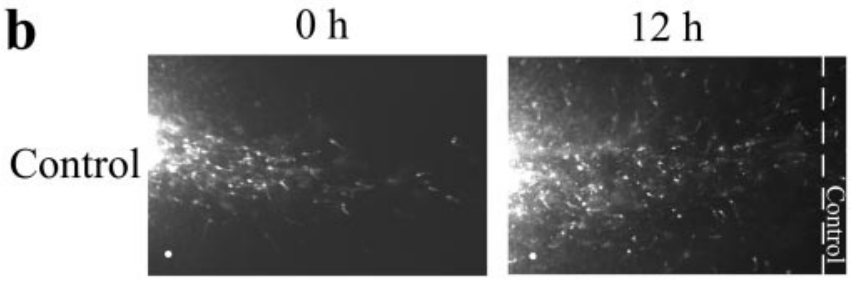

Slit
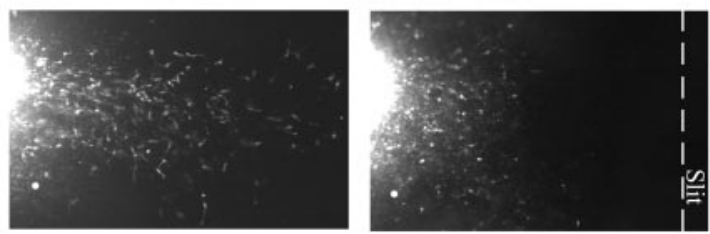

Figure 4. Neuronal repulsion by Slit in situ. a, Diagram of the experimental design. Rostral is at the right, and dorsal is at the top of the diagram. The pathway taken by SVZa neurons into the olfactory bulb is marked by green arrows. Sagittal sections of P3-P5 rat brains were made using a vibratome. Dil crystals were placed at the caudal portion of the RMS, brain sections were cultured for $12 \mathrm{hr}$ before either control cell aggregates or Slit-secreting cell aggregates were placed in the most rostral portion of the RMS, and slices were cultured for another $12 \mathrm{hr}$. The boxed region indicates the area of photography shown in $b . b$, Pictures of the same brain slice before $(0 \mathrm{hr})$ and after $(12 \mathrm{hr})$ aggregate placement are shown. The dashed lines represent the edges of the aggregates, and the white dots represent stationary points on the slice.

aggregate placement $(0 \mathrm{hr})$ and after aggregate placement $(12 \mathrm{hr})$. Whereas the number of labeled proximal neurons more than doubled after control aggregate placement, the number of labeled proximal neurons after Slit aggregate placement was halved (mean 12:0 hr HEK ratio $=2.47$; mean 12:0 hr Slit ratio $=0.47$; $n=4$ slices/group; two-sample $t$ test; $p<0.05$ ). These results indicate that Slit can function as a repellent for neurons migrating in their native pathways.

\section{Neurons migrate back into the SVZa explants in the presence of Slit in the medium}

To ascertain whether high concentrations of Slit can immobilize migrating neurons, we used a time-delayed culture assay to test the effect of Slit presented in the culture medium on neurons migrating out of the SVZa explants (Fig. 5). SVZa explants were cultured for $24 \mathrm{hr}$ before conditioned media from either control or Slit-secreting HEK cells were applied to the medium in which the SVZa explants were cultured. As expected, the number of neurons surrounding the explants continued to increase after treatment with control media (Fig. $5 a-c$ ) (two-sampled $t$ test; $p<0.005 ; n=15$ explants). However, there was a statistically significant reduction in the number of neurons surrounding explants after exposure to Slit media compared with the number of neurons before treatment (Fig. $5 a-c$ ) (two-sampled $t$ test; $p<$ $0.005 ; n=15$ explants). This reduction in cell number was not attributable to cell death or decreased cell viability, because neurons migrated normally after the removal of the conditioned media (Fig. 5a, right). Surprisingly, exposure of explants to a uniform application of Slit caused neurons to reverse their course and to migrate back into the explants (Fig. 5e). This directional reversal was observed in neurons undergoing chain migration (Fig. $5 e$, arrowhead) as well as in those migrating without contact with other cells (Fig. $5 e$, arrow). Removal of the explant and replacement with collagen/matrigel immediately before treatment with conditioned medium completely blocked Slit-induced migration of these neurons to the original sites of the explants (data not shown).

To rule out the possibility that the concentration of Slit used in our studies is lower than the concentration used in previous studies, suggesting immobilization, we tested our Slit media in the traditional culture assay (with Slit media applied from the onset of SVZa explant cultures). We found that these Slit media resulted in an almost complete absence of neuronal migration out of the explant (Fig. $5 d$ ). Furthermore, time-lapse recording indicates that Slit did not immobilize the SVZa neurons (Fig. 5e). Therefore, we conclude that previous findings suggesting that Slit can immobilize migrating neurons (Mason et al., 2001) may be attributable to migration of neurons back into explants in the presence of Slit.

\section{Reduction of the speed of migratory neurons by a uniform concentration of Slit}

Although the above findings indicate that Slit cannot immobilize migrating neurons, we addressed whether Slit had any effect on migratory speed when presented uniformly in the media. Explants were cultured for $24 \mathrm{hr}$ to allow individual neurons to migrate out of the explants. Because we noticed that the presence of an explant caused cells to turn around after uniform Slit application (Fig. 5), we performed the remainder of these experiments in the absence of an explant. After removal of the explants, timelapse microscopy was performed. The speed of individual neurons was first measured in the presence of control conditioned media, and then the speed of these same neurons was measured in the presence of either control conditioned media or Slit conditioned media. The addition of Slit caused a small but reproducible and statistically significant reduction in migratory speed (paired $t$ test; $p<0.0005 ; n=210$ ) (Fig. $6 a, b$ ). In contrast, the addition of control media did not cause a statistically significant change in migratory speed (paired $t$ test; $p=0.275 ; n=215$ ) (Fig. $6 a, b)$.

\section{Neuronal repulsion by Slit in the absence of concurrent reduction in cell speed}

Although the results from Figure 6 indicate that Slit has the potential to inhibit neuronal migration, we determined whether inhibition and repulsion of neuronal migration are always paired effects of Slit. To address this question, we used the same experimental approach described in Figure 3 and measured the speed of individual neurons before the addition of Slit and after Slitinduced turning (Fig. 7a). Neurons that responded to Slit were analyzed for their migratory speed. If repulsion and inhibition are always paired effects of Slit, one would predict that neurons would migrate more slowly after Slit-induced turning than before Slit application. However, no significant change in cell speed was observed after turning (paired $t$ test; $p=0.731 ; n=24$ ) (Fig. $7 c$ ). Furthermore, when the speed of individual neurons is plotted (Fig. $7 b$ ), it was observed that some neurons increased their speed, whereas others decreased their speed after turning away from the source of Slit. These results indicate that there are conditions in which Slit causes only repulsion, and not inhibition, of migrating neurons, suggesting a primarily repulsive role for Slit. 

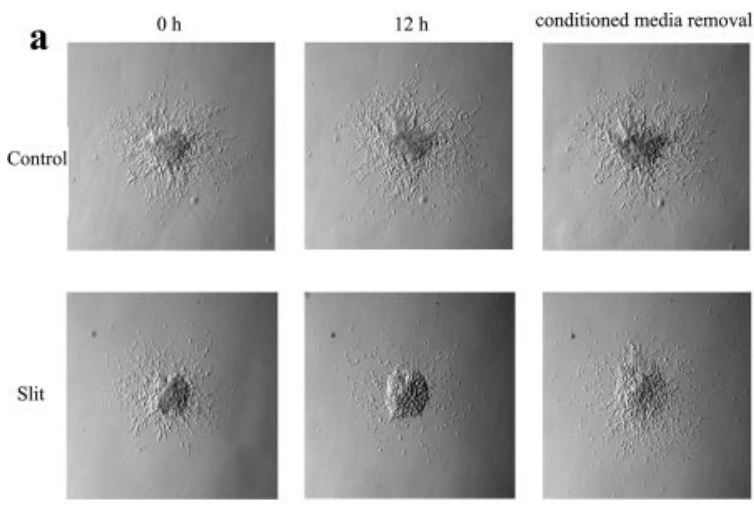

d
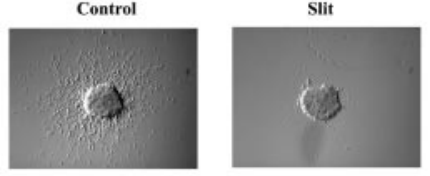

e
$0 \mathrm{~h}$

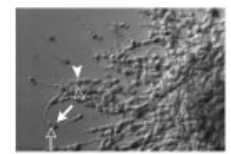

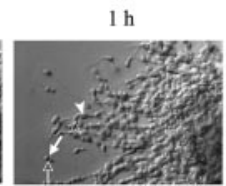

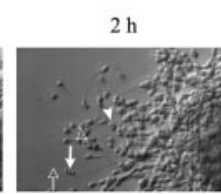

b

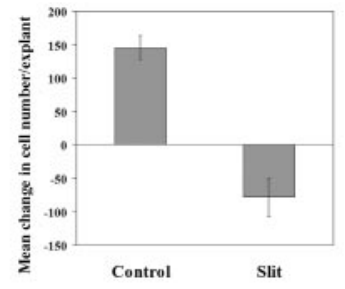

c
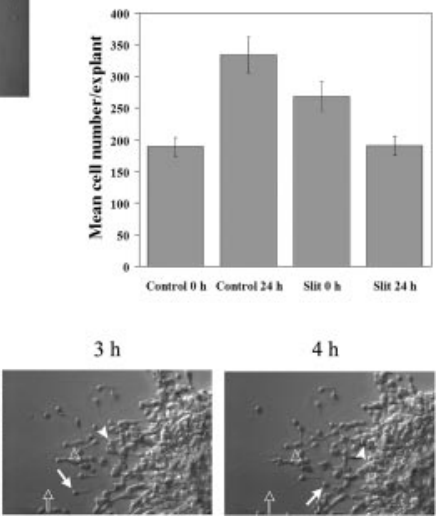

Figure 5. Turning of neurons back into SVZa explants when Slit is present in the medium. $a$, Explants were cultured for $24 \mathrm{hr}$ in the absence of exogenous factors and then cultured in the presence of Slit or control conditioned medium for $12 \mathrm{hr}$. After the incubation, the conditioned media were replaced with normal culture media and explants were cultured for an additional $16 \mathrm{hr}$. The same explant is shown in both series of pictures. b, c, Quantification of Slit effect. Explants were photographed immediately before the addition of conditioned medium and $24 \mathrm{hr}$ after the addition of conditioned medium. At both time points, the number of neurons surrounding each explant was counted ( $n=15$ explants in each group). Error bars represent SEM. $d$, The same media used in $a-c$ completely prevent migration of neurons out of the explants when supplied from the beginning of the cocultures. Explants were cultured either in control conditioned medium or in Slit conditioned medium for $24 \mathrm{hr}$ and photographed. $e$, Time-lapse microscopy showing neuronal migration back into the explants after Slit application in the media. Both neurons engaged in chain migration (arrowhead), and isolated cell migration (arrow) turned around after the addition of a uniform application of Slit and returned to the explant. Open arrows and arrowheads mark the starting positions of these two neurons in each frame.

\section{Discussion}

Our results have provided conclusive evidence that Slit is a repellent for migrating neurons when supplied from a point source. In addition, Slit can reduce the speed of, but not immobilize, migratory neurons when present in a uniform concentration. However, Slit can also function as a repellent for neurons in the absence of any concurrent reduction in cell speed, indicating that the primary effect of Slit on neuronal migration is repulsive. These studies demonstrate the importance of directly investigating the effects of specific molecules on cell motility and migratory direction and provide a solid basis for understanding the roles of Slit and other guidance cues in the developing nervous system and their use in specific therapeutic settings. The approaches designed here are useful and generally applicable assays for distinguishing between repulsion and inhibition or attraction and promotion.

Distinction between repulsion and inhibition has functional implications in how a molecule controls neuronal migration in the developing and adult nervous systems. The three mammalian Slit genes are expressed in a dynamic pattern in the nervous system (Yuan et al., 1999; Marillat et al., 2002). If Slit is solely an inhibitor, it would mean that regions expressing Slit will be nonpermissive to neurons expressing the Roundabout (Robo) receptors. In a special scenario, when neurons are generated in regions that also make Slit, these neurons will be immobilized and, thus,
$4 \mathrm{~h}$

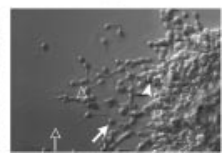

stay in such regions. If Slit is a repellent, it can guide these neurons to targets away from the source of Slit. In the scenario in which neurons are generated in regions that also make Slit, these neurons will move away from such regions. Our findings of a primarily repulsive effect of Slit indicate that responsive neurons will be directed by regions expressing Slit to move to target regions. The effect of Slit in reducing the migratory speed of, but not immobilizing, neurons indicates that responsive neurons will not be attached to Slit-expressing regions in the nervous system.

Although it has been shown that Slit affects the behavior of migrating neurons (Hu, 1999; Wu et al., 1999; Zhu et al., 1999; Mason et al., 2001), the question of whether Slit functions as an inhibitor or as a repellent has not been answered conclusively. Previous studies of Slit function have relied on traditional coculture assays in which Slit aggregates were cultured next to SVZa explants throughout the duration of the experiment ( $\mathrm{Hu}, 1999$; $\mathrm{Wu}$ et al., 1999; Zhu et al., 1999; Hirata et al., 2001; Mason et al., 2001; Wong et al., 2001). In each case, it was found that coculture of SVZa explants with Slit aggregates resulted in asymmetric distribution of neurons at the end point of the experiment. However, the shortcoming of such experiments is that changes in neuronal distribution could be caused either by Slit-induced inhibition or by Slit-induced repulsion. In the case of inhibition, a gradient of Slit emanating from the aggregate could impede migration of cells to a greater extent on the proximal side of the SVZa explant than on the distal side of the SVZa explant, resulting in asymmetric distribution. In the case of repulsion, neurons would migrate equally out of both proximal and distal sides of the SVZa explants, but the gradient of Slit would cause neurons on the proximal side of the explant to turn around and migrate to the distal side of the explant. Previous experiments using brain slice RMS migration assays have similar shortcomings (Wu et al., 1999; Zhu et al., 1999). In fact, it can be argued that all traditional coculture assays, whether they attempt to address repulsion or attraction, have the inherent problem in that they fail to distinguish between changes in cell motility versus changes in migratory direction. This argument can be applied to studies investigating cell migration as well as axon guidance. In axon guidance studies, the turning assay with Xenopus spinal cord axons is the defining assay for directional guidance (Song et al., 1998). However, the Xenopus turning assay is timeconsuming and difficult to establish, and results can vary sometimes because they are obtained from recordings of individual neurons dissociated from the spinal cord. Our assays of delayed coculture and time-lapse microscopy can provide conclusive evidence concerning the roles of secreted molecules in directional guidance and, at the same time, are technically simpler than pipette assays. An additional advantage over pipette assays is that the results from all neurons (rather than only subpopulations) can be recorded and are quite consistent. 
$\mathbf{a}$

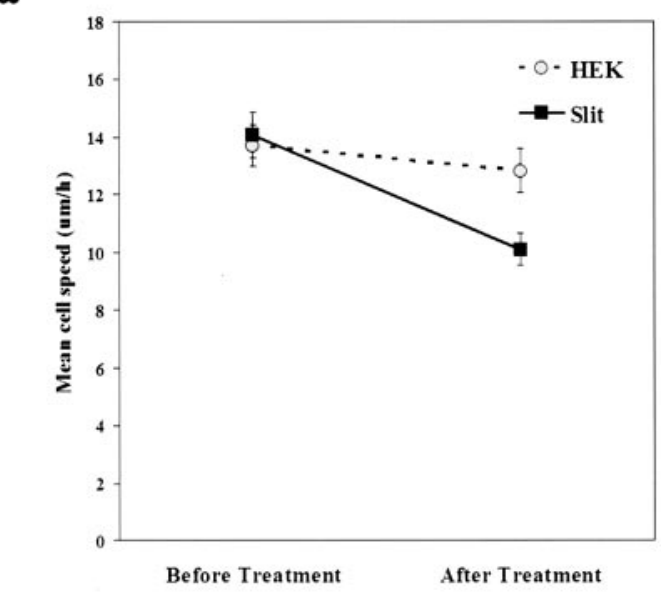

b

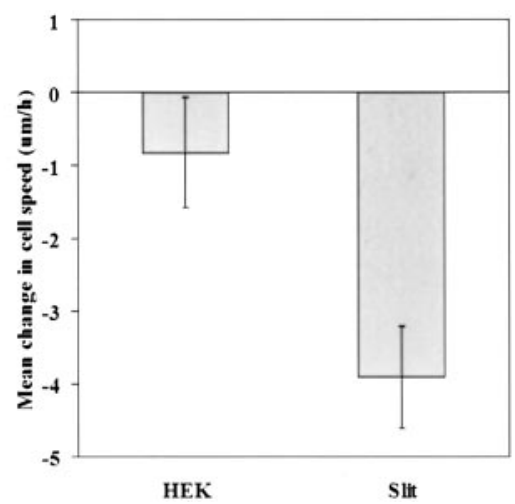

Figure 6. Reduction of migratory speed by Slit supplied in a uniform concentration. Explants were cultured for $24 \mathrm{hr}$ before recording. Explants were then removed, and the migration of individual neurons was recorded for $5 \mathrm{hr}$ in the presence of control conditioned medium, followed by another $5 \mathrm{hr}$ in the presence of either Slit conditioned medium or control conditioned medium. The individual neuronal speeds from two independent sets of experiments were pooled and averaged. Error bars represent SEM.

It was reported recently that Slit does not have the properties of a repellent but, in fact, functions solely as an inhibitor of migrating SVZa neurons (Mason et al., 2001). However, these authors argue that Slit functions as an inhibitor of cell migration, because fewer cells migrate out of SVZa explants in the presence of Slit. In the course of our studies, we noticed that the application of Slit in a uniform concentration caused migrating neurons to change direction (but not become immobilized) and to return to the explant from which they originated (Fig. 5). Our findings indicate that the primary reason for the previously observed reduction in neuronal number is that Slit causes neurons to migrate into the explants. There are several possible explanations for this observation. Although Slit concentration is uniform in the medium, there could be a concentration difference between the medium and the explant if Slit diffusion is limited in the explant, (i.e., Slit is at its lowest concentration in the center of the explant). This difference may then direct SVZa neurons toward the explant. A secondary effect of Slit could also account for such an observation and could be attributable to a number of possibilities. First, Slit may induce the explant to produce a factor that attracts SVZa neurons. Second, Slit may cause SVZa neurons to change or enhance their response to a constitutively secreted, but previously unidentified, attractant secreted by the explant. Third, a

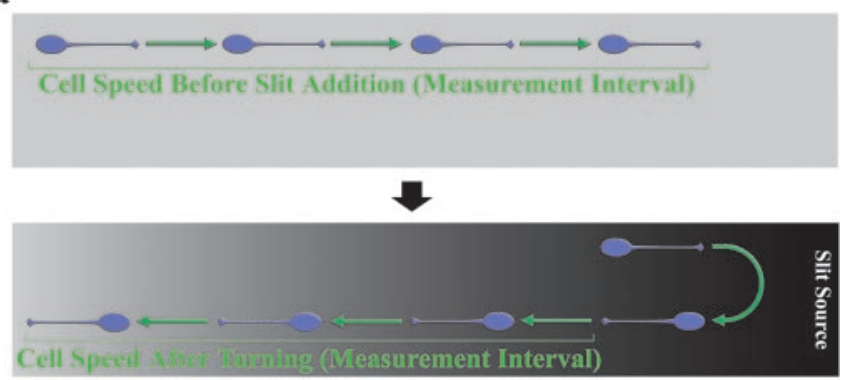

b

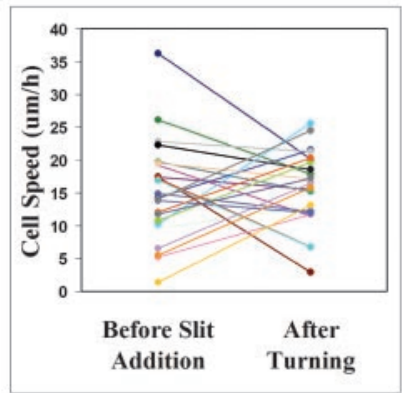

c

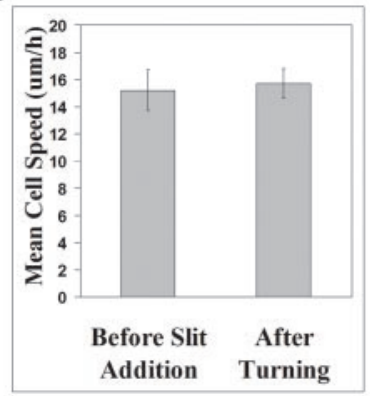

Figure 7. Repulsion of neurons by Slit without a concurrent reduction in cell speed. Timelapse microscopy of migrating neurons before and after Slit aggregate placement was performed as described in Figure 3. $a$, Diagram defining the measurement periods of neuronal speed before the addition of Slit and after Slit-induced turning. Speed measurements after turning were not begun until after neurons actually moved away from the Slit source. $b$, Individual neuronal speeds before the addition of Slit and after turning. Each neuron is designated by a different color. $c$, Mean neuronal speed before the addition of Slit and after turning $(n=$ 24). Error bars represent SEM.

Slit may be processed by the explant itself, changing Slit from a repellent to an attractant and forming an attractant microgradient.

We found that uniform application of Slit caused a small decrease in the speed of neuronal migration. However, the biological relevance of this effect still needs to be addressed, especially in light of our finding that neurons can be repelled by Slit without a concurrent reduction in the migratory speed. In fact, the finding of reduced speed while in the presence of a uniform concentration of Slit can be interpreted as being caused by the underlying mechanism of repulsion and not by a true biological role of Slit. A popular model of the general mechanism of repulsion holds that repulsive cues cause local inhibition on the cellular level that in turn leads to directed cell migration. For example, protrusion of the side of the cell closest to the repellent source is inhibited more than the side of the cell farthest away, leading to preferential protrusion of the cell on the less-inhibited side. If this is the mechanism governing repulsion, uniform application of a repellent should act as a general inhibitor of cell migration, because it inhibits protrusion of the cell in all directions. This has not been observed in our explant experiments or brain slice cultures. We note that although the use of explant culture in both collagen gels and brain slices covers a range of possible diffusion rates, we nonetheless do not know the precise concentrations of Slit in vivo or the gradient of Slit in vivo or in vitro.

Our demonstration of Slit-mediated repulsion of neurons in situ indicates that appropriate in vivo placement of Slit can theoretically be used to direct transplanted neurons or regenerated neurons to the desired sites after brain injuries or neurodegeneration, thus establishing the principle and the method for therapeutic applications of Slit in the nervous system.

In leukocytes, it has been shown that Slit is an inhibitor of 
leukocyte chemotaxis (Wu et al., 2001). Because the migratory speed and morphology are different between neurons and leukocytes, it will be interesting to investigate whether Slit is primarily an inhibitor or both an inhibitor and repellent of leukocyte migration.

\section{References}

Bagri A, Marin O, Plump AS, Mak J, Pleasure SJ, Rubenstein JL, TessierLavigne M (2002) Slit proteins prevent midline crossing and determine the dorsoventral position of major axonal pathways in the mammalian forebrain. Neuron 33:233-248.

Bashaw GJ, Goodman CS (1999) Chimeric axon guidance receptors: the cytoplasmic domains of slit and netrin receptors specify attraction versus repulsion. Cell 97:917-926.

Bashaw GJ, Kidd T, Murray D, Pawson T, Goodman CS (2000) Repulsive axon guidance: Abelson and Enabled play opposing roles downstream of the roundabout receptor. Cell 101:703-715.

Brose K, Bland KS, Wang KH, Arnott D, Henzel W, Goodman CS, TessierLavigne M, Kidd T (1999) Slit proteins bind Robo receptors and have an evolutionarily conserved role in repulsive axon guidance. Cell 96:795-806.

Colamarino SA, Tessier-Lavigne M (1995) The role of the floor plate in axon guidance. Annu Rev Neurosci 18:497-529.

Flanagan JG, Vanderhaeghen P (1998) The ephrins and Eph receptors in neural development. Annu Rev Neurosci 21:309-345.

Fricke C, Lee JS, Geiger-Rudolph S, Bonhoeffer F, Chien CB (2001) Astray, a zebrafish roundabout homolog required for retinal axon guidance. Science 292:507-510.

Hatten ME, Heintz N (1998) Neurogenesis and migration. In: Fundamentals of neuroscience (Zigmond M, ed), pp 451-479. New York: Academic.

Hatten ME, Mason CA (1990) Mechanisms of glial-guided neuronal migration in vitro and in vivo. Experientia 46:907-916.

Hirata T, Fujisawa H, Wu JY, Rao Y (2001) Short-range guidance of olfactory bulb axons is independent of repulsive factor slit. J Neurosci 21:2373-2379.

Hu H (1999) Chemorepulsion of neuronal migration by Slit2 in the developing mammalian forebrain. Neuron 23:703-711.

Hutson LD, Chien CB (2002) Pathfinding and error correction by retinal axons: the role of astray/robo2. Neuron 33:205-217.

Kidd T, Bland KS, Goodman CS (1999) Slit is the midline repellent for the robo receptor in Drosophila. Cell 96:785-794.

Klein R (2001) Excitatory Eph receptors and adhesive ephrin ligands. Curr Opin Cell Biol 13:196-203.

Kolodkin AL (1996) Growth cones and the cues that repel them. Trends Neurosci 19:507-513.

Li HS, Chen JH, Wu W, Fagaly T, Zhou L, Yuan W, Dupuis S, Jiang ZH, Nash W, Gick C, Ornitz DM, Wu JY, Rao Y (1999) Vertebrate slit, a secreted ligand for the transmembrane protein roundabout, is a repellent for olfactory bulb axons. Cell 96:807-818.

Lois C, Alvarez-Buylla A (1994) Long-distance neuronal migration in the adult mammalian brain. Science 264:1145-1148.

Luskin MB (1993) Restricted proliferation and migration of postnatally generated neurons derived from the forebrain subventricular zone. Neuron 11:173-189.

Marillat V, Cases O, Nguyen-Ba-Charvet KT, Tessier-Lavigne M, Sotelo C, Chedotal A (2002) Spatiotemporal expression patterns of slit and robo genes in the rat brain. J Comp Neurol 442:130-155.

Mason HA, Ito S, Corfas G (2001) Extracellular signals that regulate the tangential migration of olfactory bulb neuronal precursors: inducers, inhibitors, and repellents. J Neurosci 21:7654-7663.

Nguyen Ba-Charvet KT, Brose K, Marillat V, Kidd T, Goodman CS, TessierLavigne M, Sotelo C, Chedotal A (1999) Slit2-mediated chemorepulsion and collapse of developing forebrain axons. Neuron 22:463-473.

Nüsslein-Volhard C, Wieschaus E, Kluding H (1984) Mutations affecting the pattern of the larval cuticle in Drosophila melanogaster. I. Zygotic loci on the second chromosome. Rouxs Arch Dev Biol 193:267-282.

Plump AS, Erskine L, Sabatier C, Brose K, Epstein CJ, Goodman CS, Mason CA, Tessier-Lavigne M (2002) Slit1 and Slit2 cooperate to prevent premature midline crossing of retinal axons in the mouse visual system. Neuron 33:219-232.

Rakic P (1990) Principles of neural cell migration. Experientia 46:882-891. Raper JA (2000) Semaphorins and their receptors in vertebrates and invertebrates. Curr Opin Neurobiol 10:88-94.

Rice DS, Curran T (2001) Role of the Reelin signaling pathway in central nervous system development. Annu Rev Neurosci 24:1005-1039.

Ross ME, Walsh CA (2001) Human brain malformations and their lessons for neuronal migration. Annu Rev Neurosci 24:1041-1070.

Rothberg JM, Artavanis-Tsakonas S (1992) Modularity of the slit protein. Characterization of a conserved carboxy-terminal sequence in secreted proteins and a motif implicated in extracellular protein interactions. J Mol Biol 227:367-370.

Rothberg JM, Hartley DA, Walther Z, Artavanis-Tsakonas S (1988) slit: an EGF-homologous locus of D. melanogaster involved in the development of the embryonic central nervous system. Cell 55:1047-1059.

Song HJ, Ming GL, He Z, Lehmann M, Mckerracher L, Tessier-Lavigne M, Poo M-M (1998) Conversion of neuronal growth cone responses from repulsion to attraction by cyclic nucleotides. Science 281:1515-1518.

Stein E, Tessier-Lavigne M (2001) Hierarchical organization of guidance receptors: silencing of netrin attraction by slit through a Robo/DCC receptor complex. Science 291:1928-1938.

Wang KH, Brose K, Arnott D, Kidd T, Goodman CS, Henzel W, TessierLavigne M (1999) Biochemical purification of a mammalian slit protein as a positive regulator of sensory axon elongation and branching. Cell 96:771-784.

Whitford KL, Marillat V, Stein E, Goodman CS, Tessier-Lavigne M, Chedotal A, Ghosh A (2002) Regulation of cortical dendrite development by SlitRobo interactions. Neuron 33:47-61.

Wong K, Ren XR, Huang YZ, Xie Y, Liu G, Saito H, Tang H, Wen L, BradyKalnay SM, Mei L, Wu JY, Xiong WC, Rao Y (2001) Signal transduction in neuronal migration: roles of GTPase activating proteins and the small GTPase Cdc42 in the Slit-Robo pathway. Cell 107:209-221.

Wong K, Park HT, Wu JY, Rao Y (2002) Slit proteins: guidance cues for cells ranging from neurons to leukocytes. Curr Opin Genet Dev 12:583-591.

Wu JY, Feng L, Park H-T, Havlioglu N, Wen L, Tang H, Bacon KB, Jiang Z, Zhang X-C, Rao Y (2001) The neuronal repellent Slit inhibits leukocyte chemotaxis induced by chemotactic factors. Nature 410:948-952.

Wu W, Wong K, Chen JH, Jiang ZH, Dupuis S, Wu JY, Rao Y (1999) Directional guidance of neuronal migration in the olfactory system by the secreted protein Slit. Nature 400:331-336.

Yuan W, Zhou L, Chen JH, Wu JY, Rao Y, Ornitz DM (1999) The mouse SLIT family: secreted ligands for ROBO expressed in patterns that suggest a role in morphogenesis and axon guidance. Dev Biol 212:290-306.

Zhu Y, Li HS, Zhou L, Wu JY, Rao Y (1999) Cellular and molecular guidance of GABAergic neuronal migration from the striatum to the neocortex. Neuron 23:473-485.

Zhu Y, Yu T, Zhang X-C, Nagasawa T, Wu JY, Rao Y (2002) Role of the chemokine SDF-1 as the meningeal attractant for embryonic cerebellar neurons. Nat Neurosci 5:719-720. 